\title{
Clinical implications of mechanisms of action of antidepressants
}

\author{
Brian Leonard
}

The purpose of this review is briefly to consider the biological basis of depression and to attempt to illustrate how antidepressants produce their beneficial effects by correcting these abnormalities.

\section{Biochemical changes in patients with depression and mechanisms of action of antidepressant drugs}

In 1965, Schildkraut postulated that noradrenaline may play a pivotal role in the aetiology of depression. In favour of this hypothesis was the observation that the antihypertensive drug reserpine, which depletes central and peripheral vesicular stores of catecholamines such as noradrenaline, can precipitate depression in patients in remission. The experimental drug $\alpha$-methyl-paratyrosine that blocks the synthesis of noradrenaline and dopamine by inhibiting the rate-limiting enzyme tyrosine hydroxylase also precipitates depression in patients during remission. Such findings are only indirect indicators that noradrenaline plays an important role in human behaviour, and may be defective in depression - more direct evidence is needed to substantiate the hypothesis. The most obvious approach would be to determine the concentration of noradrenaline and / or its major central metabolite 3-methoxy-4-hydroxyphenylglycol (MHPG) in the brains of suicide victims. The problem with such post-mortem studies is that: (a) the precise diagnosis may be uncertain;

(b) there is usually a considerable post-mortem delay before the brain is removed at autopsy; and

(c) suicide is often committed by taking an overdose of alcohol together with drugs, which grossly affects central monoamine neurotransmitter function.

Unless these variables are carefuly controlled, the value of results obtained from such analyses is uncertain. The neurotransmitter receptors in post-mortem brain are less labile than the neurotransmitters that act upon them. The density of $\beta$-adrenoceptors is increased in cortical regions of the brains from suicide victims who have suffered from depression. There is also an increase in the density of $\beta$-adrenoceptors on the lymphocytes of untreated patients with depression. As the density of these receptors is normalised by effective antidepressant treatment, it has been postulated that changes in the $\beta$-receptor density may be a state marker of the condition (Leonard, 1994a).

Elevation of growth hormone in the plasma following administration of the $\alpha 2$ adrenoceptor agonist clonidine is diminished in patients with depression, which suggests that central post-synaptic $\alpha 2$ adrenoceptors are also sub-functional in such patients. As the clonidine response does not return to normal after effective antidepressant treatment, this is possibly a trait marker of depression.

In depression, it should be emphasised that the reduced growth hormone response to clonidine cannot be accounted for by the drug treatment, age or gender of the patient, which supports the view that the noradrenergic system is dysregulated.

Brian E. Leonard obtained his PhD in neuropharmacology at the University of Birmingham in 1962 and his DSc from the National University of Ireland in 1976. His major research interest is in psychopharmacology. Since 1974 he has been Professor of Pharmacology at the National University of Ireland (Pharmacology Department, National University of Ireland, Galway, Ireland). 
Box 1. Changes in brain and tissue amine neurotransmitters in patients with depression which may be indicative of the mechanism of action of antidepressants

Evidence from the brains of patients with depression who committed suicide

Increased density of $\boldsymbol{\beta}$-adrenceptors in cortical regions

Increased density of $5-\mathrm{HT}_{2 \mathrm{a}}$ receptors in limbic regions

Decreased concentration of 5-HIAA in several brain regions

Increased muscarinic receptor density in limbic regions

Lastly, determination of the urine or plasma concentrations of MHPG (an indicator of central noradrenergic activity), suggests that central noradrenergic function is sub-optimal in depression. Taken together, these results suggest that central noradrenergic function is decreased in depression, an event leading to an increase in the density of the post-synaptic $\beta$-adrenoceptors (Leonard, 1986; Dinan, 1994). However, none of the studies of noradrenergic function in post-mortem material or tissues from patients with depression is entirely satisfactory. Many of the findings cannot be replicated, the number of patients studied is relatively small and the tritiated ligands used to determine the receptor density, for example, vary in their selectivity.

The density of muscarinic receptors is also increased in limbic regions of patients with depression who have commited suicide. If such a change reflects an increased activity of the cholinergic system, it could reduce noradrenergic function, as increased central cholinergic activity can precipitate depression and reduce noradrenergic activity (Janowsky et al, 1986).

The role of serotonin (5-hydroxytryptamine, 5-HT) has also been extensively studied in patients with depression. Whereas the overall psychophysiological effects of noradrenaline in the central nervous system appear to be linked to drive and motivation, 5-HT is primarily involved in the expression of mood (see Charney et al, 1991). The main 5-HT metabolite, 5-hydroxyindole acetic acid (5-HIAA), is reduced in the cerebrospinal fluid (CSF) of patients with severe depression, as are 5-HT and 5-HIAA in the limbic regions of the brain of suicide victims (Agren, 1980). Serotonin receptor function is also abnormal in depression with an increase in the density of cortical $5-\mathrm{HT}_{2 \mathrm{a}}$ receptors in the brains of suicide victims and also on the platelet membrane of patients with depression.

Platelets are, like neurons, of ectodermal origin and contain enzymes such as enolase that are otherwise restricted to neurons. In addition, platelets contain storage vesicles for 5-HT, from which the amine is released by a calcium-dependent mechanism. An energy-dependent transport site for 5-HT also occurs on the platelet membrane, the structure of which is identical to that found on neurons in the brain. Furthermore, the platelet membrane contains $5-\mathrm{HT}_{2 \mathrm{a}}$ and $\alpha 2$ adrenergic receptors that are functionally involved in platelet aggregation; there is evidence that the densities of these receptors are increased in patients with depression and largely normalised following effective treatment. The transport of ${ }^{3} \mathrm{H}-5-\mathrm{HT}$ into the platelet is significantly reduced in untreated patients with depression, but largely returns to normal following effective treatment (Leonard, 1994b). This change, occurring irrespective of the nature of the antidepressant, may be a state marker of the illness.

The function of the 5- $\mathrm{HT}_{2 \mathrm{a}}$ receptor appears subnormal in depression, as shown by diminished aggregatory response to 5-HT in vitro, but normalises when the patient recovers. The number of $5-\mathrm{HT}_{2 \mathrm{a}}$ receptors on the platelet membrane of patients with depression is increased, a finding which suggests that the G-protein and second messenger phosphatidyl inositol system is defective. Also, the modulatory site on the 5-HT transporter, the imipramine binding site, is decreased in depression, but remains unchanged by effective treatment. This suggests that the number of imipramine binding sites on the platelet membrane is a trait marker of the condition. However, the binding of the more specific ligand, ${ }^{3} \mathrm{H}$-paroxetine, is unchanged in depression. It is uncertain how the changes in platelet function reflect those occurring in the brain. The changes observed could be a reflection of the hormonal changes (for example, glucocorticoids) that occur in depression. Some of the alterations in platelet function are a consequence of low molecular weight plasma factors that reduce following effective antidepressant treatment (Nugent \& Leonard, 1998).

Patients undergoing treatment with selective serotonin reuptake inhibitors (SSRIs) rapidly relapse when given an amino acid-containing drink that is deficient in tryptophan (Delgado et al, 1990). The onset of the symptoms of depression is rapid (less than 24 hours); the reduction in brain serotonin as a consequence of the lack of tryptophan may be the cause of the altered mood state. However, those patients with depression in this study who responded to desipramine, which enhances 
Box 2. Other clincial studies implicating an abnormal biogenic amine function in depression

Decreased 5-HIAA concentration in CSF

Decreased homovanillic acid (main dopamine metabolite) in CSF

Decreased urinary excretion of the main central noradrenaline metabolite MHPG(?)

Rapid relapse following administration of a tryptophan-free amino acid drink to patients with depression being treated with an SSRI (?)

Rapid relapse following administration of the tyrosine hydroxylase inhibitor $\alpha$-methyltyrosine to patients with depression who respond to a noradrenaline reuptake inhibitor such as desipramine

?, finding equivocal

noradrenergic function, did not relapse when given the tryptophan-free drink. Some patients with depression have a pronounced deficit in noradrenergic function rather than serotonergic function, which may account for the differential response of some patients with depression to antidepressants that augment the serotonergic or noradrenergic system.

\section{Dopaminergic function}

Studies on platelets, lymphocytes, changes in cerebrospinal fluid metabolites of brain monoamines and post-mortem studies suggest that a major abnormality in both noradrenergic and serotonergic function occurs in depression, and that such changes could be causally related to the disease process.

Less attention has been paid to the possible involvement of dopamine in this disorder. However, anhedonia is a characteristic feature of major depression, and a defect in dopaminergic function is thought to be causally involved in this symptom (Willner, 1983). The concentration of the main dopamine metabolite, homovanillic acid (HVA), is decreased in the CSF of patients with depression, particularly those with psychomotor retardation. Patients with depression who attempted suicide were also found to have a decreased urinary excretion of HVA and the second major dopamine metabolite, dihydroxyphenylacetic acid (DOPAC). The dopamine deficit may be a reflection of the degree of retardation rather than of the psychological state, as similar changes in CSF HVA concentrations have been reported to occur in patients with Parkinson's disease. Changes in the basal ganglia may overshadow any changes in the mesolimbic dopaminergic system, as the contribution of this area is relatively minor.

Bupropion (not marketed in Europe but available in North America) amineptine and nomifensin (withdrawn because of the rare occurrence of haemolytic anaemia) owed their antidepressant efficacy to their ability to increase central dopaminergic function. There are also open-label studies to suggest that the novel dopamine receptor agonist roxindole and the selective dopamine uptake inhibitor pramipexole may have antidepressant action.

\section{Is there a common mechanism of action?}

\section{Why is there a delay in onset of the antidepressant response?}

Delay in onset of the therapeutic effect of antidepressants, which clearly is unrelated to the acute actions of these drugs on monoamine reuptake transporters or monoamine oxidase, is probably related to the adaptive changes that occur in the pre- and post-synaptic receptors that govern neurotransmitter release.

Antidepressant action usually has a gradual onset of over 2-3 weeks before the optimal effect is obtained (World Health Organization Mental Health Collaborating Centres, 1989). Much of the

Box 3. Evidence from patients with depression

Increased density of 5- $\mathrm{HT}_{2 \mathrm{a}}$ receptors on the platelet membrane

Decreased uptake of ${ }^{3} \mathrm{H}-5-\mathrm{HT}$ into platelet

Increased $\beta$-adrenoceptor density on lymphocyte membrane

Increased density of $\alpha 2$ adrenoceptors on platelet membanes(?)

Blunted growth hormone response to a clonidine challenge

Blunted prolactin response to a fenfluramine challenge 
Box 4. Changes in neurotransmitter receptors that occur in the cortex of rat brain following chronic antidepressant treatment

Cortical $\beta$-adrenoceptors:

Cortical $\alpha 1$ adrenoceptors:

Cortical $\alpha 2$ autoreceptors:

Dopamine autoreceptors:

Cortical GABA-B receptors:

Limbic 5-HT $\mathrm{H}_{\mathrm{a}}$ receptors:

Cortical 5- $\mathrm{HT}_{2 \mathrm{a}}$ receptors: decreased functional activity and density

increased density

decreased functional activity

decreased functional activity

decreased density

increased density and decreased functional activity

increased density and decreased functional activity

N.B. Most of these changes have been reported to occur with different classes of antidepressants but only following chronic treatment

early improvement is probably associated with a reduction in anxiety and improvement in sleep caused by the sedative action of the drugs. Delay in onset of the therapeutic response cannot be easily explained by the pharmacokinetic profile of the drugs, as peak plasma (and presumably brain) concentrations are usually reached in 7-10 days. Furthermore, the 2- to 3-week delay is also seen in many patients given electroconvulsive therapy (ECT). Box 4 summarises some of the changes in neurotransmitter receptors that occur in the cortex of rat brain following ECT or the chronic administration of antidepressants. Adaptational changes occur in adrenoceptors, serotonin, dopamine and type B $\gamma$-aminobutyric acid (GABA-B) receptors (Leonard, 1994a). GABA-B receptors play a role in enhancing noradrenaline release in the cortex and in this respect differ fundamentally from the inhibitory GABA-A receptors that facilitate central GABAergic transmission. A decrease in the activity of GABA-B receptors may therefore contribute to the reduced central noradrenergic tone in depression.

\section{Changes in cholinergic function}

In the bulbectomised rat model of depression, a decrease in cortical muscarinic receptors occurs that returns to control values following treatment with antidepressants (Earley et al, 1994).

The anticholinergic activity of the tricyclic antidepressants is usually associated with their unacceptable peripheral side-effects and most second-generation antidepressants lack such sideeffects. Possible support for a cholinergic hypothesis of depression is that the cholinesterase inhibitor pyridostigmine, when administered to drug-free patients with depression, causes an enhanced activation of the anterior pituitary glands, as shown by the release of growth hormone ( $\mathrm{O}^{\prime}$ Keane et al, 1992). This suggests that the muscarinic receptors are supersensitive in patients with depression. Janowsky et al (1986) postulated that depression arises as the result of an imbalance between the central noradrenergic and cholinergic systems; in depression, the activity of the former system is decreased and, conversely, in mania it is increased. As most antidepressants enhance noradrenergic function, it is hypothesised that the reduction in cholinergic activity is a consequence of the increase in noradrenergic activity.

In summary, irrespective of the specificity of the antidepressnts following their acute administration, it can be speculated that a common feature of all of

Box 5. Changes in cholinergic and aminergic receptors in depression and following antidepressant treatment

Evidence that central muscarinic receptors are supersensitive in patients with depression and that chronic antidepressant treatments normalise this supersensitvity. This effect does not depend on any intrinsic anticholinergic activity of the antidepressant (i.e. it is an indirect, adaptive effect)

Following chronic administration to rats, there is evidence that most antidepressants cause adapative changes in $5-\mathrm{HT}_{1 \mathrm{a}^{\prime}}$ $5-\mathrm{HT}_{2 \mathrm{a}^{\prime}} \alpha 1, \alpha 2$ and $\beta$-adrenoceptors, GABA-B receptors and possibly the $N$ methyl-D-aspartate (NMDA)-glutamate receptors 
these drugs is to correct the abnormality in neurotransmitter receptor function. Such an effect of chronic antidepressant treatment may parallel the time of onset of the therapeutic response.

\section{Link between the serotonergic and noradrenergic systems}

Chronic administration of antidepressants enhances the inhibitory response of forebrain neurons to microiontophoretically applied 5-HT. This enhanced response is blocked by lesions of the noradrenergic projections to the cortex. This dual effect could help to explain enhanced serotonergic function that arises after chronic administration of antidepressants or ECT. Conversely, impairment of serotonergic function by means of selective neurotoxins (e.g. 5,7-dihydroxytryptamine) or a 5-HT synthesis inhibitor (e.g. parachlorophenylalanine) largely prevents the decrease in functional activity of cortical $\beta$-adrenoceptors that usually arises following chronic antidepressant treatment. 5- $\mathrm{HT}_{1 \mathrm{~b}}$ receptors are located on serotonergic nerve terminals and act as autoreceptors; stimulation by serotonin decreases the further release of this amine. Chronic administration of selective SSRIs slowly desensitises the inhibitory $5-\mathrm{HT}_{1 \mathrm{~b}}$ autoreceptors and thereby enhances serotonin release.

Likewise, the $5-\mathrm{HT}_{1 \mathrm{a}}$ somatodendritic receptors inhibit the release of serotonin and it is posulated that the enhanced release of the transmitter following chronic administration of SSRIs is a consequence of adaptive down-regulation of the inhibitory $5-\mathrm{HT}_{1}$ receptors (Blier et al, 1990). The validity of this hypothesis is supported by the pharmacological effect of 5- $\mathrm{HT}_{1 \mathrm{a}}$ antagonists. Thus, the beta-blocker and $5-\mathrm{HT}_{1 \mathrm{a}}$ antagonist pindolol, in combination with fluoxetine or paroxetine, may enhance the therapeutic efficacy of the SSRI and, in some studies, reduce the time to onset of the peak therapeutic effect (Tome et al, 1997). However, several investigators have not been able to replicate such findings (see McAskill et al, 1998).

Serotonin can also regulate dopamine turnover. A positive correlation exists in patients with depression between the HVA and 5-HIAA concentrations in the CSF (Agren, 1980). Stimulation of the 5 -HT cell bodies in the median raphe causes reduced firing of the substantia nigra, where dopamine is the main neurotransmitter. Serotonin plays an important role in modulating dopaminergic function in many regions of the brain, including the mesolimbic system. The effects of some antidepressants that show an apparent selectivity for the serotonergic system could be ascribed to secondary changes in dopaminergic pathways.

\section{Role of the glutamatergic system in the action of antidepressants}

The glutamatergic system is the main excitatory neurotransmitter pathway in the brain. Tricyclic antidepresssants (TCAs) inhibit the binding of dizolcipine to the ion channel of the main glutamate receptor, the NMDA-receptor in the brain (Reynolds \& Miller, 1988). Newer antidepressants have a qualitatively similar effect (Kitayama et al, 1997). Whether this is due to direct action on glutamate receptor sites or indirect action via the glycine receptor site is uncertain. Glycine and drugs modulating the glycine site show antidepressantlike activity in animal models of depression. Thus, antidepressants may act as functional NMDA receptor antagonists.

\section{Intracellular changes that occur following chronic antidepressant treatment}

The coupling of the NDMA-receptor on the cell surface to the intracellular second messenger is brought about by a member of the G-protein family.

Box 6. Mechanism of action of antidepressants: changes in serotonergic function

There is experimental evidence that the chronic administration of antidepressants or ECT enhances the inhibitory effect of microiontophoretically applied 5-HT. This effect is blocked by lesions of the noradrenergic projections to the frontal cortex

SSRIs after chronic administration downregulate the inhibitory $5-\mathrm{HT}_{1 \mathrm{a}}$ receptors on the serotonergic cell body, thereby leading to an enhanced release of the transmitter from the nerve terminal

Serotonin can also decrease dopamine release from the substantia nigra (an important dopaminergic nucleus). This may account for the observation that some SSRIs cause dystonias and precipitate the symptoms of parkinsonism if given to such patients who are responding to L-dopa. Sertraline appears to differ from other SSRIs in this respect and may slightly enhance dopaminergic function by reducing reuptake of this transmitter 
Beta-adrenoceptors are linked to adenylate cyclase and, depending on the subtype of receptors, 5-HT is linked to either adenylate cyclase $\left(5-\mathrm{HT}_{1 \mathrm{a}}, 5-\mathrm{HT}_{1 \mathrm{~b}}\right)$ or phospholipase $\left(5-\mathrm{HT}_{2 \mathrm{a}^{\prime}} 5-\mathrm{HT}_{2 \mathrm{c}}\right)$. Activation of phospholipase results in an intracellular increase in the secondary messengers diacylglycerol and inositol triphosphate (IP3), the IP3 then mobilising intraneuronal calcium.

The net result of the activation of the secondary messenger systems is to increase the activity of the various intracellular protein kinases (termed third messengers) that phosphorylate cell proteins to produce a physiological response. Racagni et al (1991) have investigated the effect of chronic antidepressant treatment on the phosphorylation of proteins associated with the cytoskeletal structure of the nerve cell. Antidepressants could affect the function of the cytoskeleton by changing the component of the associated protein phosphorylation system. Thus, both typical (e.g. desipramine) and atypical antidepressants (e.g. (+)oxaprotiline, a specific noradrenaline reuptake inhibitor, and fluoxetine, an SSRI) increased the synthesis of a microtubule fraction. These changes occurred only after chronic antidepressant treatments. Antidepressants might thereby change neuronal signal transduction processes distal to the receptor.

\section{Glucocorticoid receptors: adaptive changes following antidepressant treatment}

Glucocorticoid receptors have been identified in the nuclei of catecholamine and 5-HT-containing cell bodies in the brain. Glucocorticoid receptors activate deoxyribonucleic acid-binding proteins, which can modify the transcription of genes. Chronic administration of imipramine increases glucocorticoid receptors in rat brain, particularly the noradrenergic and serotonergic cell body regions.

Lymphocyte glucocorticoid receptors are subsensitive in patients with depression. The failure of the negative feedback mechanism that regulates the secretion of adrenal glucocorticoids further suggests that the central glucocorticoid receptors are subsensitive. Hypersecretion of cortisol is a characteristic feature of many patients with major depression (Dinan, 1994). Central neurotransmission occurring in depression may, in part, result from the effects of chronic glucocorticoids.

Glucorticoid synthesis inhibitors (e.g. metyrapone) may be able to reduce the abnormality in neurotransmitter function by decreasing the cortisol concentration.

Typical antidepressants increase the density of glucocorticoid receptors in rats. Such an effect could
Box 7. Possible role of excitatory amino acids and intracellular second messengers in the action of antidepressants

In experimental studies, chronic antidepressant treatments have been shown to reduce the behavioural effects of the NMDA-glutamate receptor antagonist dizolcipine. This suggests that antidepressants may act as functional NMDA receptor antagonists and thereby reduce excitatory glutamate transmission that is mediated by NMDA receptors

Intracellular protein phosphorylation is enhanced by chronic antidepressant treatment. This leads to the increased synthesis of microtubules that form an important feature of the cellular cytoskeleton. Thus, antidepressants might change signal transduction within the neuron

Enhanced synthesis and transport of neurotransmitter-synthesising enzymes (e.g. tyrosine and tryptophan hydroxylase)

increase the negative feedback mechanism and thereby reduce the release of cortisol. In support of this hypothesis, there is preliminary clinical evidence that metyrapone (and the steroid synthesis inhibitor ketoconazole) may have antidepressant effects. Recently, several lipophilic antagonists of corticorophin-releasing factor (CRF) type 1 receptor, which appears to be hyperactive in the brain of patients with depression, have been shown to be active in animal models of depression. Clearly, this is a potentially important area for antidepressant development (Mansbach et al, 1997).

Glucocorticoid receptors are present in high density in the amygdala, and neuroimaging studies have shown that the amygdala is the only structure in which the regional blood flow and glucose metabolism consistently correlate positively with the severity of depression (Lesser et al, 1994). This hypermetabolism appears to reflect an underlying pathological process as it also occurs in asymptomatic patients and in the close relatives of patients.

\section{Effects of antidepressants on endocrine-immune functions}

Stress is frequently a trigger factor for depression in vulnerable patients. Corticorophin-releasing factor is elevated in the CSF of untreated patients with 
depression, which presumably leads to the hypercortisolaemia that usually accompanies the condition. One of the consequences of elevated plasma glucocorticoids is a suppression of some aspects of cellular immunity. Many aspects of both cellular (for example, natural killer cell activity, Tcell replication) and non-cellular (for example, raised acute phase proteins) immunity are abnormal in untreated patients with depression (see Song \& Leonard, 1995). This may contribute to the susceptibility of patients with depression to physical ill health.

A link between CRF, the cytokines that orchestrate many aspects of cellular immunity and the prostaglandins of the E series has been the subject of considerable research in recent years. Prostaglandin E2 (PGE2) concentrations are raised in the plasma of untreated patients with depression and are normalised following effective treatment with TCAs. Raised PGE2 concentrations reflect increased proinflammatory cytokines (particularly interleukins 1 and 6 and tumour necrosis factor, ), which occur as a consequence of increased macrophage activity in the blood and brain. In the brain, the microglia function as macrophages and produce such cytokines locally. Thus, the increased synthesis of PGE2 may contribute to the reduction in amine release in the brain that appears to underly the pathology of depression. It has recently been postulated that antidepressants normalise central neurotransmission by reducing brain concentrations of both the cytokines and PGE2 by inhibiting central and peripheral macrophage activity together with cyclooxygenase type 2 activity in the brain. Cyclooxygenase is the key enzyme in the synthesis of the prostaglandins. The usefulness of TCAs in severe rheumatoid arthritis may reflect the inhibitory action of such drugs on cyclooxygenase activity. These effects of TCAs, together with those in glucocorticoid receptor function, may thus normalise defective central neurotransmission.

Box 8. Role of glucocorticoids in modulating brain amines in depression

Glucocorticoid receptors occur on catecholamine- and 5-HT-containing cell bodies in the brain. There is evidence that the glucocorticoid receptors are hyposensitive in patients with depression. Chronic antidepressant treatment sensitises these receptors, thereby normalising the noradrenergic and serotonergic function that is reduced by hypercortisolaemia, which occurs in major depression

\section{Antidepressants and changes in neuronal structure}

Another possible mechanism whereby antidepressants may change the physical relationship between neurons in the the brain is by inhibiting neurite outgrowth from nerve cells. Amitriptyline inhibits neurite outgrowth from chick embryonic cerebral explants in vivo (Wong et al, 1991). A common mode of action of all antidepressants could be to modify the actual structure of nerve cells and possibly eliminate inappropriate synaptic contacts that are responsible for behavioural and psychological changes associated with depression.

It is also possible that chronic antidepressant treatment may affect pathways that involve receptor interactions with protein tyrosine kinases, by increasing specific growth factor synthesis or by regulating the activity of proinflammatory cytokines (Duman et al, 1997). These pathways control many aspects of neuronal function that ultimately underlie the ability of the brain to adapt and respond to environmental stimuli. The infusion of one of these transcription factors (brain-derived neutrophic factor) into the mid-brain of rats results in antidepressant-like activity (Siuciak et al, 1996), an action associated with an increase in the synthesis of tryptophan hydroxylase, the rate limiting enzyme in the synthesis of serotonin.

\section{Conclusions}

Circumstantial evidence from the body fluids of patients, and from the brains of suicide victims who suffered from depression, indicate an abnormality

Box 9. The possible role of prostaglandins and cytokines in depression

There is evidence that both cellular and noncellular immunity are abnormal in patients with depression. The proinflammatory cytokines (interleukins 1 and 6 and tumour necrosis factor alpha) from macrophages are raised in depression. This leads to increased prostaglandin E2 synthesis and release, which may lead to a reduction in central monoamine release. Chronic antidepressant treatments reduce both the proinflammatory cytokines and prostaglandin E2 
in serotonergic and noradrenergic function (together with changes in other transmitters such as acetylcholine and GABA). Such changes in amine neurotransmitter function generally normalise following effective antidepressant treatment. It is postulated that all types of antidepressants and ECT, irrespective of their presumed specificity for acting on a particular neurotransmitter system, ultimately correct the multitude of neurotransmitter abnormalities that cause depression.

My personal view is that these changes in neutrotransmitters, which may be causally connected to the symptoms of depression, are secondary to those involving hyposensitive central glucocorticoid receptors, proinflammatory cytokines and an increase in prostaglandin E2 (both centrally and peripherally), together with changes in the structure of neurons. Thus, factors such as the duration of antidepressant treatment and delay in onset of the clinical response may reflect the subcellular adaptive changes that underlie the mechanism of action of these drugs.

\section{References}

Agren, H. (1980) Symptom patterns in unipolar and bipolar depression correlating with monoamine metabolities in the cerebrospinal fluid: II. Suicide. Psychiatric Research, 3, 225-236.

Blier, P., de Montigny, C. \& Chaput, Y. (1990) A role for the serotonin system in the mechanism of action of antidepressant treatments: preclinical evidence. Journal of Clinical Psychiatry, 51, 14-20.

Charney, D. S., Delgado, P. L., Price, L. H., et al (1991) The receptor sensitivity hypothesis of antidepressant action. In The Role of Serotonin in Psychiatric Disorders (eds S.-L. Brown \& H. M. van Praag), pp. 27-56. New York: Brunner/Mazel. Delgado P. L., Charney, D. S., Price, L. H., et al (1990) Serotonin function and the mechanism of antidepressant action. Reversal of antidepressant induced remission by rapid

Box 10. Changes in neuronal structure in depression

There is evidence that inadequately treated or untreated major depression is associated with a decrease in hippocampal volume. This could be a consequence of the increase in proinflammatory cytokines and hypercortisolaemia

Experimental evidence suggests that chronic antidepressant treatments increase the formation of transcription factors within the brain, which increases neuronal plasticity and leads to recovery depletion of plasma tryptophan. Archives of General Psychiatry, 47, 411-418.

Dinan, T. G. (1994). Glucocorticoids and the genesis of depressive illness. A psychobiological model. British Journal of Psychiatry, 164, 365-371.

Drevets, W. C. (1998) Functional neuroimaging studies of depression: the anatomy of melancholia. Annual Review of Medicine, 49, 341-361.

Duman, R. S., Henninger, G. R. \& Nestler, E. J. (1997) A molecular and cellular theory of depression. Archives of General Psychiatry, 54, 597-606.

Earley, B., Glennon, M., Lally, M., et al (1994) Autoradiographic distribution of cholinergic nuscarinic receptors and serotonin ${ }^{2}$ receptors in olfactory bulbectomized (OB) rats after chronic treatment with mianserin and desipramine. Human Psychopharmacology, 9, 397-407.

Janowsky, D. S., Risch, S. C., Kennedy, B., et al (1986) Central muscarinic effects of physostigmine on mood, cardiovascular function, pituitary and adrenal neuroendocrine release. Psychopharmacology, 89, 150-154.

Kitayama, I., Yaga, T., Kayahara, T. J., et al (1997) Longterm stress degenerates, but imipramine regenerates, noradrenergic axons in the rat cerebral cortex. Biological Psychiatry, 42, 687-696.

Leonard, B. E. (1986) Neurotransmitter receptors, endocrine response and the biological substrates of depression: a review. Human Psychopharmacology, 1, 3-18.

- (1994a) Biological strategies for the development of antidepressants. CNS Drugs, 1, 285-304.

- (1994b) Effect of antidepressants on specific neurotransmitters: are such effects relevant to their therapeutic action? In Handbook of Depression and Anxiety - A Biological Approach (eds J. A. den Boer \& J. M. A. Sitzen), pp. 379404. New York: Marcel Dekker.

Lesser, I. M., Mena, I. \& Boone K. B. (1994) Reduction of cerebral blood flow in older depressed patients. Archives in General Psychiatry, 51, 677-686.

Mansbach, R. S., Brooks, E. N. \& Chen, Y. L. (1997) Antidepressant-like effects of CP-154, 526, a selective CRF receptor antagonist. European Journal of Pharmacology, 323, 21-26.

McAskill, R., Mir, S. \& Taylor, D. (1998) Pindolol augmentation of antidepressant therapy. British Journal of Psychiatry, 173, 203-208.

Nugent, D. F. \& Leonard, B. E. (1998) Platelet abnormalities in depression. Journal of Serotonin Research, 4, 251-266.

O'Keane, V., O'Flynn, K., Lucey. J., et al (1992) Pyridostigmine induced growth hormone responses in healthy and depressed subjects - evidence for cholinergic supersensitivity in depression. Psychological Medicine, 22, 55-60.

Racagni, G., Tinelli, D. \& Bianchi, E. (1991) cAMP dependent binding proteins and endogneous phosphorylation after antidepressant treatment. In Hydroxytryptamine in Psychiatry (eds M. Sandler, A. Coppen \& S. Hartnet) (5th edn), pp. 116-123. Oxford: Oxford Medical Publications.

Reynolds, I. J. \& Miller, R. J. (1988) Tricyclic antidepressants block $N$-methyl-D-aspartate receptor: similarities to the action of zinc. British Journal of Pharmacology, 95, 95-102.

Siuciak, J. A., Lewis, D., Wiegand, S. J., et al (1996) Antidepressant like effect of brain derived neutrophic factor. Pharmacology, Biochemistry and Behavior, 56, 131137

Song, C. \& Leonard, B. E. (1995) The effect of olfactory bulbectomy in the rat, alone or in combination with antidepressants on immune function. Human Psychopharmacology, 10, 7-18.

Tome, M. B., Isaac M. T., Harte, R., et al (1997) Paroxetine and pindolol: a randomized trial of serotonergic autoreceptor blockade in the reduction of antidepressant latency. International Clinical Psychopharmacology, 12, 81-89.

World Health Organization Mental Health Collaborating Centres (1989) Pharmacotherapy of depressive disorders: a consensus statement. Journal of Affective Disorders, 17, 197-198.

Wong, K. L., Bruch, R. C. \& Farbman, A. I. (1991) Amitriptyline-mediated inhibition of neurite outgrowth 
from chick embryonic cerebral explants involves a reduction in adenylate cyclase activity. Journal of Neurochemistry, 57, 1223-1230.

Willner, P. (1983) A conceptual framework for psychobiological synthesis. Biological Psychiatry, 18, 1447-1450.

\section{Multiple choice questions}

1. Compared with controls, the following have been suggested as biological markers of depression:

a reduced plasma cortisol after $1 \mathrm{mg}$ dexamethasone treatment

$\mathrm{b}$ reduced growth hormone secretion after clonidine treatment

c reduced prolactin secretion after fenfluramine treatment

$\mathrm{d}$ decreased 5-HIAA concentration in the CSF.

2. The following changes have been reported to occur on platelet membranes of patients with depression:

a increased ${ }^{3} \mathrm{H}$-paroxetine binding to the 5-HT transporter

b decreased ${ }^{3} \mathrm{H}$-imipranine binding to the 5-HT transporter

c increased density of $\beta$-adrenoceptors

$\mathrm{d}$ decreased density of $5-\mathrm{HT}_{2 \mathrm{a}}$ receptors.
3. The following mechanisms have been postulated to account for the therapeutic action of antidepressants following their chronic administration: a decreased density (down-regulation) of cortical $\beta$-adrenoceptors

b increased density (up-regulation) of cortical $\beta$-adrenoceptors

c decreased density of $\alpha 2$ (inhibitory) adrenoceptors in limbic regions of the brain

$\mathrm{d}$ desentitisation of inhibitory $5-\mathrm{HT}_{1 \mathrm{~b}}$ receptors in limbic regions of the brain.

MCQ answers

123

a $\mathrm{F} \quad$ a $\mathrm{F}$ a $\mathrm{T}$

$\begin{array}{lll}\text { b } T & \text { b } T & \text { b }\end{array}$

$\begin{array}{lll}\text { c } T & \text { c } F & \text { c }\end{array}$

d $\mathrm{T} \quad \mathrm{d} \mathrm{F} \quad$ d $\mathrm{T}$

e $F \quad$ e $F \quad$ e $F$

\section{American Psychiatric Association Annual Meeting}

13-17 May 2000

Chicago

The Royal College of Psychiatrists and BMJ Publishing will be promoting their books and journals together at this important congress. The on-line versions of the three College journals, British Journal of Psychiatry, Advances in Psychiatric Treatment and Psychiatric Bulletin, will be launched at this meeting. The journal Evidence-Based Mental Health is currently available on-line and will also be demonstrated during the congress.

We look forward to welcoming you to our stand at the

\section{Publishers Bookfair, booth number 323.}

The Royal College of Psychiatrists, 17 Belgrave Square, London SW1X 8PG Telephone +44 (0)20 7235 2351; fax +44 (0)20 72596507

\section{http://www.rcpsych.ac.uk}

